\title{
Parallel Compressive Sampling Matching Pursuit Algorithm for Compressed Sensing Signal Reconstruction with OpenCL
}

\author{
Fang Huang ${ }^{a, b, c}$, Jian $\mathrm{Tao}^{c}$, Yang Xiang ${ }^{d}$, Peng Liu $^{e}$, Lei Dong ${ }^{a}$ and Lizhe Wang ${ }^{e, f, \dagger}$ \\ ${ }^{a}$ School of Resources $\mathcal{E}$ Environment, University of Electronic Science and Technology of China, Chengdu 611731, P. R. China \\ ${ }^{b}$ Institute of Remote Sensing Big Data, Big Data Research Center, University of Electronic Science and Technology of China, Chengdu 611731, P. R. China \\ ${ }^{c}$ Center for Computation E Technology, Louisiana State University, LA 70803, USA \\ ${ }^{d}$ School of Information Technology, Deakin University, Victoria 3216, Australia \\ ${ }^{e}$ Institute of Remote Sensing and Digital Earth, Chinese Academy of Sciences, Beijing 10094, P. R. China \\ ${ }^{f}$ School of Computer Science, China University of Geosciences, Wuhan 430074, P. R. China \\ ${ }^{\dagger}$ Corresponding author, Lizhe.Wang@computer.org
}

\begin{abstract}
Compressive sensing (CS) is a new signal processing method, which was developed recent years. CS can sample signals with a frequency far below the Nyquist frequency. CS can also compress the signals while sampling, which can reduce the usage of resources for signal transmission and storage. However, the reconstruction algorithm used in the corresponding decoder is highly complex and computationally expensive. Thus, in some specific applications, e.g., remote sensing image processing for disaster monitoring, the CS algorithm usually cannot satisfy the time requirements on traditional computing platforms. Various studies have shown that many-core computing platforms such as OpenCL are among the most promising platforms that are available for real-time processing because of their powerful floating-point computing capabilities. In this study, we present the design and implementation of parallel compressive sampling matching pursuit (CoSaMP), which is an OpenCL-based parallel CS reconstruction algorithm, as well as some optimization strategies, such as access efficiency, numerical merge, and instruction optimization. Based on experiments using remote sensing images with different sizes, we demonstrated that the proposed parallel algorithm can achieve speedups of about 41 times and 58 times on AMD HD7350 and NVIDIA K20Xm platforms, respectively, without modifying the application code.
\end{abstract}

Key words: Compressive sensing, CoSaMP, Heterogeneous computing, Many-core computing, OpenCL

\section{Introduction}

Compressive sensing (CS) is a revolutionary signal acquisition and reconstruction technique, which can allow signal sampling at a rate far below the Nyquist sampling rate, and it can reconstruct the original signal with a numerical optimization algorithm $[1,2]$. CS has been applied widely in many traditional image processing areas, such as image denoising, image compression, and image fusion [3]. Subsequently, it has also been employed for processing remote sensing (RS) images [4? ]. However, many challenges still need to be addressed before applying this method to real-world RS applications because RS images are usually multi-temporal, with high resolution and large volumes. Thus, the traditional serial CS algorithm, which only runs on a single CPU, is not sufficient for some specific $\mathrm{RS}$ applications that require real-time processing [5].

Image reconstruction is the major component of the overall CS theory. Many image reconstruction algorithms are available, such as orthogonal matching pursuit (OMP) and compressive sampling matching pursuit (CoSaMP), but in most cases, the procedures used by these reconstruction algorithms are timeconsuming. Moreover, the widespread adoption of multi-core and many-core computing platforms means that parallelization is essential for speeding up computationally intensive applica- tions $[6,7,8]$. Thus, many different parallel approaches have been proposed for accelerating the processing of the reconstruction algorithm.

- For example, in a study using multi-core CPUs, Wu et al. divided the coefficients obtained after sparse transformation into several sub-images using interleaving extraction, before assigning these sub-images to each thread on a multi-core CPU using OpenMP, thereby improving the image reconstruction performance. The speedup obtained in their study was up to 7.35 times compared with a singlethread CPU [9]. Zhang et al. also developed an OMP parallel algorithm using OpenMP, which could reduce the execution time by $60-70 \%$ using a quad-core CPU compared with the single-thread operation, where the image size was $256 \times 256$ [10]. However, the performance improvements obtained using the methods of $\mathrm{Wu}$ et al. and Zhang et al. are limited by the scheduling of the assembly pipeline in the operating system and the computing capacity of the CPU.

- Based on hardware parallelism, Yang et al. designed an OMP parallel algorithm using a field-programmable gate array (FPGA), which could recover $97.8 \%$ of the origi- 
nal signal and it had a high processing efficiency [11]. In addition, Chen et al. developed a parallel reconstruction algorithm using an FPGA + CPU + graphical processing unit (GPU) hybrid mode, which achieved a speedup of 13 times [12]. However, this approach has limitations such as the complexity of the design and implementation, while it also requires a good understanding of the hardware architecture as well as the corresponding programming framework, which are major barriers to use by scientists.

In recent years, due to their powerful computing capabilities, GPUs have provided major breakthroughs in parallel computing performance [13]. For example, the Chinese Tianhe-1A supercomputer, which is one of the largest GPU-enabled supercomputers, has a peak computing rate of 4700 TFLOPS and it was the fastest computer in the world from October 2010 to June 2011. GPU-based computing has performance advantages in terms of data processing and it has been used widely in some data-intensive and computing-intensive applications $[15,16 ?$ ? ]. Many researchers have studied the used of CS algorithms on GPU platforms, e.g., Alexandre et al. implemented a parallel CS Moreau-Yosida regularization algorithm on a GPU platform [17]; Andrecut et al. implemented the parallel matching pursuit (MP) algorithm [18]; Yong et al. designed and implemented OMP using the compute unified device architecture (CUDA) and obtained a speedup of up to 40 times compared with serial algorithm processing [19]; Geng et al. proposed the iterative shrinkage-threshold algorithm by using a CUDA programming model based on a GPU, which improved the sparse microwave imaging speed [20]; and Blanchard et al. also implemented a series of parallel reconstruction algorithms for CS, such as iterative hard thresholding, hard thresholding pursuit, and CoSaMP [21]. In addition, Chen et al. used the CS algorithm to compress synthetic aperture radar images with a GPU-based platform and achieved a speedup of nearly nine times compared with that on a CPU-based platform [22].

The general-purpose GPU and programming models based on it have been used widely in CS algorithm processing, but to the best of our knowledge, most previous applications were based on the CUDA programming model, which was designed exclusively for NVIDIA GPU and it is not portable to GPU platforms supplied by other vendors [23]. Open Computing Language (OpenCL) is an open industry standard for heterogeneous systems and it provides a framework that allows parallel programs to be executed on various platforms. OpenCL helps to promote the more extensive usage of GPU computing [24]. In this study, we propose a parallel CoSaMP reconstruction algorithm based on the OpenCL programming model for use on a GPU-based platform. Our method differs from previous studies, where it improves the processing speed and it can also run on various GPU-based platforms without requiring modifications to the application code.

The remainder of this paper is organized as follows. Section 2 provides a brief introduction to various background topics related to the parallel reconstruction algorithm, including the CS, GPU platform, and the OpenCL program model. Section 3 describes the implementation of the parallel CoSaMP reconstruc- tion algorithm. Section 4 presents details of the experiments that we performed to verify the parallel algorithm's high performance and its cross-platform capability on different GPU-based heterogeneous platforms. Finally, in Section 5, we discuss the experimental results and give our conclusions.

\section{Introduction and Background}

\subsection{CS and the CoSaMP Algorithm}

The complete CS theory includes three main components: image sparsification, image sampling, and image reconstruction. Obtaining images with a sparse representation is the basis of CS, where the discrete cosine transform or wavelet transform (DWT) methods are typically employed for sparse processing. After sparse processing, the energy will fall into the low frequency part of the signal, i.e., the transformed signal will be sparse or close to sparse. If we assume that there is a signal $\mathrm{a} \in R^{N}$, which can be represented in a certain domain by the sparse transform matrix $\Psi$, then the transformation of $X$ will be [25]:

$$
X=\Psi a .
$$

In equation (1), $X$ has $K$ nonzero elements. Thus, $K$ is also called the sparse degree. We can then design a measurement matrix $\Phi \in R^{M * N}(\mathrm{M}<\mathrm{N})$ and obtain a measurement vector $Y \in R^{M}$, from which the measurement matrix can be recovered. This measuring process can be expressed as follows:

$$
Y=\Phi X=\Phi \Psi a
$$

or

$$
Y=\Theta a .
$$

In equation (3), the $M-b y-N$ matrix $\Theta$ is called the CS matrix and $\Theta=\Phi \Psi$. If the vector $Y$ and matrix $\Phi$ are known, $X$ can be calculated from equation (2). $X$ satisfies a system of linear equations because $M<N$, i.e., equation (2) is an under-determined system, which has no unique solution in general. However, if $X$ is sparse and $\Theta$ is subject to the restricted isometry property, then we can obtain a unique sparse solution from equation (2). The solution requires the solution of the $l_{0}$ normal optimization problem. Thus, we can reconstruct the original signal $\widehat{a}$ with a fairly high probability when it satisfies the restricted condition of $Y=\Phi \Psi \mathrm{a}$. This method can then be expressed as follows:

$$
\widehat{a}=\operatorname{argmin}\|a\|_{0} \text { s.t. } \Phi \Psi a=Y,
$$

where $\|a\|_{0}$ is the 0 -normal number for the vector, which indicates the number of nonzero elements in the vector. For simplicity, the detailed explanations of the symbols used in the equations above are described in Table 1.

However, Donoho et al. [1] noted that solving the minimum $l_{0}$ norm is an $N P$-hard problem. Thus, researchers have proposed a series of algorithms for obtaining suboptimal solutions, including basis pursuit, MP, OMP, and CoSaMP. CoSaMP is derived from the OMP algorithm, which constantly updates the index set at each iteration, and it employs the sequence code idea 
Table 1: Detailed meanings of the symbols used in the equations.

\begin{tabular}{lll}
\hline Symbols & Definition & Matrix dimension \\
\hline$a$ & Original signal & Nxl \\
\hline$\Psi$ & Sparse transform matrix & $\mathrm{NxN}$ \\
\hline $\mathrm{X}$ & Sparse transform signal & $\mathrm{Nx} 1$ \\
\hline$\Phi$ & Measurement matrix & $\mathrm{MxN}$ \\
\hline$\Theta$ & CS matrix & $\mathrm{MxN}$ \\
\hline $\mathrm{K}$ & Sparse degree & - \\
\hline $\mathrm{Y}$ & Measurement vector & $\mathrm{Mx} 1$ \\
\hline$\widehat{a}$ & Reconstructing the original signal with the & - \\
\hline$\|a\|_{0}$ & minimum normal method & \\
\hline s.t. & 0-normal number of the nonzero elements & - \\
\hline
\end{tabular}

with backtracking [26]. CoSaMP is stable and robust, where it can improve the quality of the reconstructed signal and reflect the strong anti-noise interference. The steps of the CoSaMP algorithm are listed in Table 2.

We used the performance analysis tool, Intel VTune Amplifier, to detect the hotspots in the sequential CoSaMP algorithm. Based on our analysis of the results, we found that the most time-consuming steps in the serial algorithm are Step 1, Step 4, and Step 6, which comprise $4 \%, 87 \%$, and $6 \%$ of the total time, respectively. Therefore, in order to accelerate the CoSaMP algorithm, we focused on the design and implementation of parallel approaches for these three time-consuming steps.

\subsection{GPU-based Computing}

GeForce 256 was the first general purpose GPU (GPGPU) to be designed for handling general computing tasks and it was released by NVIDIA in 1999 [27]. The GPGPU, or GPU for short, has less dependence on the CPU and it takes over some of the work from the CPU. In recent years, the development of GPU hardware has evolved at a much higher speed than that dictated by Moore's Law [28]. These improvements have greatly accelerated the speed and quality of computer graphics processing, thereby promoting the development of related fields, e.g., image processing, virtual reality, and computer simulation, as well as providing a good computational platform for processing general purpose computation tasks other than graphic processing.

There are two main GPU architectures [29]: the single instruction multiple thread (SIMT) architecture based on a scalar unit, e.g., NVIDIA's Fermi card is based on the SIMT architecture and it exhibits high flexibility and scalability; and the single instruction multiple data (SIMD) architecture based on vector unit, where the AMD Cypress architecture is a typical example of SIMD. The Cypress architecture can improve the utilization of computing resources and maximize the efficiency of scalar instructions. Both architectures are used in GPU-based parallel computing research.

\subsection{OpenCL Programming Model}

OpenCL was first proposed by the Apple Cooperation in 2008 as a framework for writing programs that can be executed on heterogeneous platforms comprising CPUs, GPUs, digital signal processors (DSPs), FPGAs, and other processors [24]. At present, OpenCL is an open standard for general-purpose computing and it is maintained by a non-profit technology consortium, the Khronos Group. It provides cross-platform compatibility and some other useful features, which can greatly facilitate software development, and promote the development of parallel applications on heterogeneous computing platforms.

OpenCL defines a C-like language for writing programs, called kernels, which are executed on the compute devices. A single compute device typically comprises many individual processing elements (PEs) and a single kernel execution can run on many or all of the PEs in parallel. In addition, OpenCL defines an application programming interface (API), which allows programs running on the host to launch kernels on the compute devices as well as managing device memory that is separate from the host memory.

A parallel algorithm will achieve better performance if suitable memory types are considered during OpenCL programming. OpenCL defines a four-level memory hierarchy for the compute device: global, constant, local, and private memory. Global memory can be shared by all of the processing elements, but it has high access latency. Constant memory is also visible to all of the compute units on the device, where it is part of the global memory. Any element of the constant memory is accessible simultaneously by all of the work-items. Local memory belongs to the compute unit and it is typically implemented on-chip, where it is shared by all of the work-items within a workgroup. It has low access latency, but its capacity is limited. Private memory belongs to a work-item and it is typically implemented on-chip in registers.

\section{Implementation of the Parallel CoSaMP Algorithm}

To improve the performance of the CoSaMP algorithm, we parallelized the time-consuming steps introduced in Section 2.1. There are two main parallelization mechanisms in 
Table 2: Steps of the CoSaMP algorithm.

Input: The measurement matrix $\Phi$, measurement vector $y$, and sparse degree $K$
Initialization: $r_{0}=y$ ( the current residual), $\widehat{x}=0(\widehat{x}$ is the estimate of $y), \Gamma^{0}=\phi$ (index set), $\mathrm{n}=1$ (iteration)
Continue loop until meeting the condition for terminating the iterations:
1) Compute signal agent, i.e., the inner product of $r$ and each column of $\Phi \cdot g^{n}=\Phi^{T} r_{n-1}(\Phi$ is Gaussian);
2) Find the best $2 K$ support set of the index set. $\Lambda=\operatorname{supp}\left(g^{n}\right)$;
3) Merge the strongest support sets. $\Gamma^{n}=\Gamma^{n-1} \cup \Lambda$. Obtain the vector $\Phi_{\Gamma^{n}}$;
4) Perform least-squares signal estimation to estimate the new value of $x . x^{n}=\left(\Phi^{T} \Gamma^{n} \Phi_{\Gamma^{n}}\right)^{-1} \Phi^{T} \Gamma^{n} \mathrm{y}$;
5) Prune signal. Compute the best $K$ support set for the signal. $\Gamma^{n}=\operatorname{supp}\left(\mathrm{x}^{n}\right), x_{k}=\Phi_{\Gamma^{n}}$;
6) Update the residual $r_{n}=\mathrm{y}-\Phi x_{k}$. Test the iteration stopping conditions: determine whether it meets $\left\|r_{n}\right\|^{2} \geq\left\|r_{n-1}\right\|^{2}$;
if it meets the condition, stop the iterations and output $\widehat{x}=x_{k}$.

OpenCL: data parallelism and task parallelism [30]. Due to the interdependency among every step in the CoSaMP algorithm, it is difficult to implement a parallel processing algorithm with multiple tasks parallelism. The data element of the CoSaMP algorithm has a weak correlation in the data structure, so it is suitable for processing independently following the data parallelism model. In this study, we employed the data parallelism approach and we designed a parallel CoSaMP algorithm to match the features of the GPU hardware. A flowchart illustrating the main process is shown in Fig.1.

From the left of Fig.1, the sequential CoSaMP algorithm mainly comprises four stages from the original image until the generation of the recovered image. First, the original image is subjected to sparse processing with the DWT. The sampling image is then obtained and the sampled image is reconstructed by image reconstitution processing. Finally, the recovered image is obtained using the IDWT operation. In this study, we focused mainly on the parallelization of the image reconstruction stage with the CoSaMP algorithm. As shown in Fig.1, it is clear that the parallel CoSaMP algorithm is divided into two parts, i.e., the CPU end and the GPU end, where the overall implementation of the parallel CoSaMP mainly comprises the following seven steps.

- Step 1: Platform initialization, memory allocation, and transferring data from the CPU to the GPU. When data are stored in the GPU memory, it is better to enforce data alignment, which needs to satisfy the requirements of combined access to improve the memory performance. If the stored data are a matrix stored in rows, it is necessary to ensure that the first address in each line is an integer multiple of the half-wavefront.

- Step 2: Compute the signal agent on the GPU by applying a matrix-vector multiplication operation in parallel.

- Step 3: Select a number of 2k largest elements from the results. The elements contain some conditional statements, so they are suitable for processing on the CPU. The results are then merged with the support index in the last cycle, and transferred from the CPU to the GPU to process the subsequent complete matrix expansion operation on the GPU in a parallel manner.
- Step 4: Signal estimation is the most time-consuming step in this algorithm, which mainly comprises a least-squares operation on GPU by Cholesky decomposition. The major procedure in this step is the implementation of parallel Cholesky decomposition operations to handle parallel matrix multiplications in an efficient manner.

- Step 5: Select $K$ largest elements from the estimation signal to complete the prune signal operation on the CPU and then copy them to the GPU.

- Step 6: Update the residual via a series of operations, i.e., matrix-vector multiplication, vector subtraction, and vector norm. During the updating processing, it is necessary to evaluate the stopping condition, where the reconstructed signal is obtained if it is satisfied; otherwise, the process will continue to the next iteration.

- Step 7: Free the relevant memories at the end of the algorithm.

According to the workflow described above, the main task performed by the parallel CoSaMP algorithm is porting the parallel implementation of some functions in the correlation steps to the GPU, such as matrix-matrix multiplication, matrix-vector multiplication, and Cholesky decomposition. The following subsections explain the parallel implementation of these functions.

\subsection{Parallel Implementation of Matrix-vector Multiplication}

It is better to use two levels in the parallel mode to improve the parallelism of matrix-vector multiplication. According to the OpenCL programming model, the parallelism of matrixvector multiplication is designed as follows. The multiplication of every row of matrix $\mathrm{A} \in R^{M * N}$ and vector $\mathrm{x} \in R^{N}$ is a coarsegrained parallelism task, which is performed best by the corresponding work-group. The product is $\left(y_{1}, y_{2}, y_{3} \ldots y_{m}\right)$, as shown in Fig.2. Vector-vector multiplication is a fine-grained parallelism problem, which should be performed by threads in the work-group, and the result is $\left(t_{1}, t_{2}, t_{3} \ldots t_{n}\right)$, as illustrated in Fig.3. To improve the efficiency of processing, we use local memory to store $\left(t_{1}, t_{2}, t_{3} \ldots t_{n}\right)$, which can be accessed by all of the threads in the work-group, thereby reducing the communication costs. 


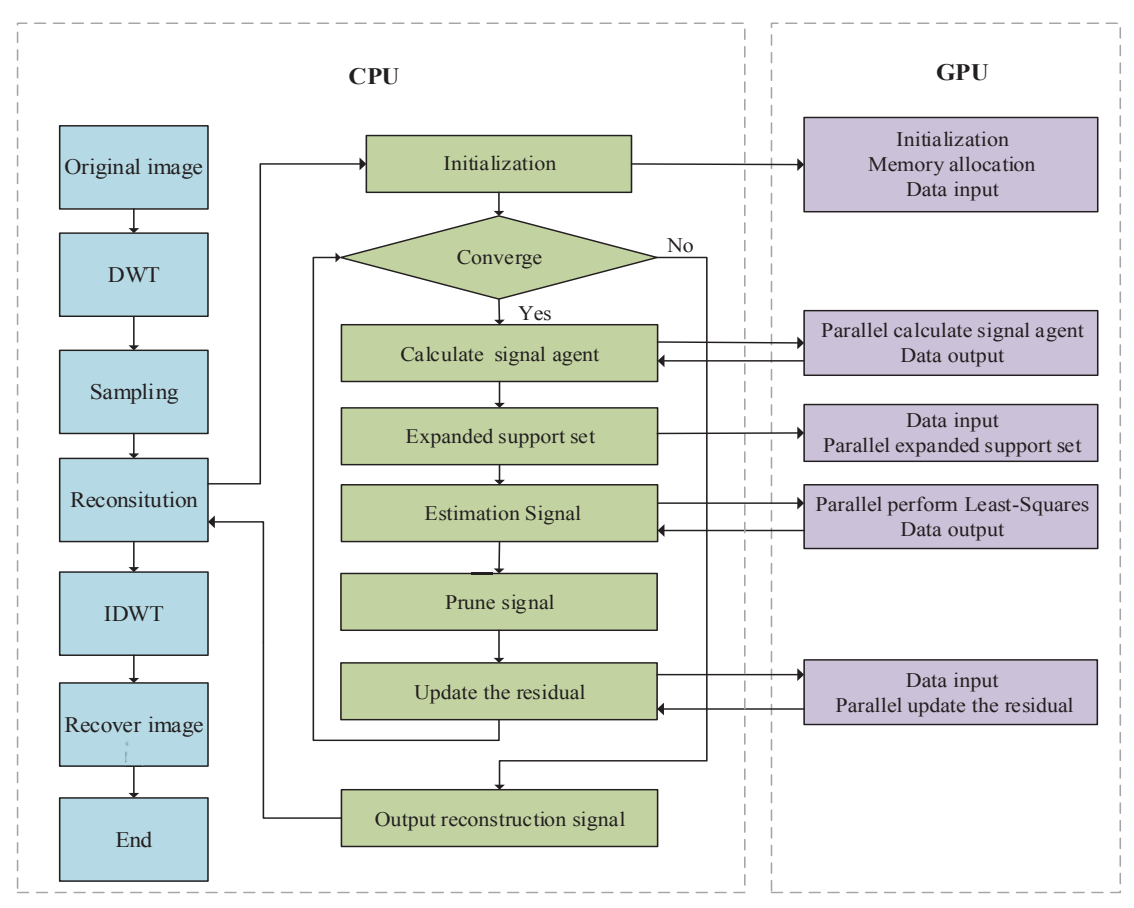

Figure 1: Parallel CoSaMP algorithm design.

The value of $y_{i}$ is the sum of $t_{i}$ stored in its corresponding workgroup. We use a numerical merging method to implement the summation and we utilize numerical merging to improve the algorithms performance, as shown in Fig.4.

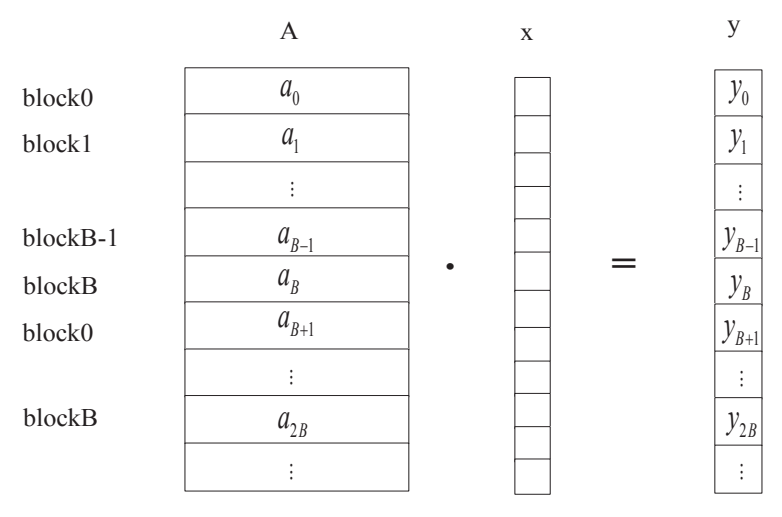

thread
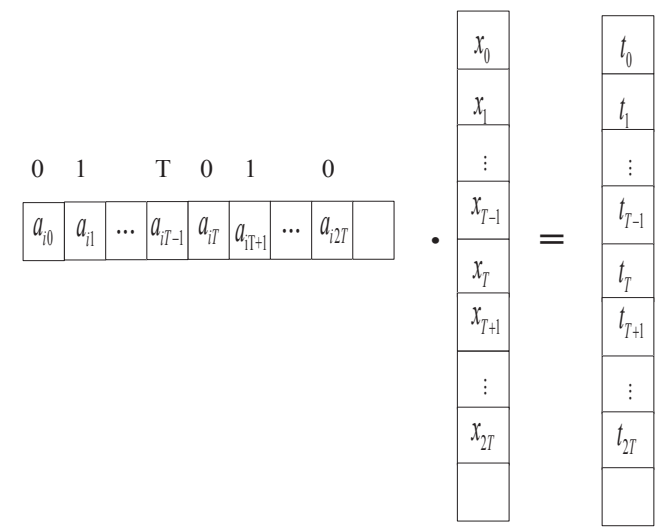

Figure 2: Coarse-grained parallelism.

Figure 3: Fine-grained parallelism.

According to the parallel design of the matrix-vector multiplication operation, the kernel function can be created, which is a function such as MatVecMul (const int m, const int n, _global const double *A, int lda, _global const double *x, _global dou- 


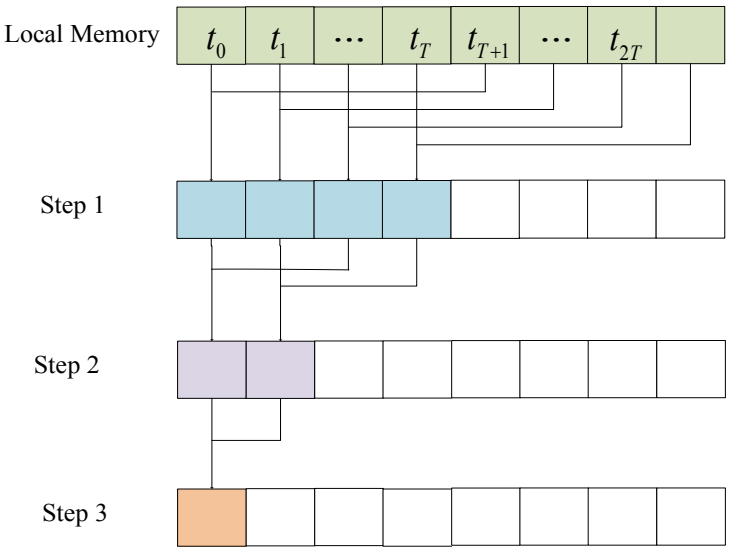

Figure 4: Numerical merging method.

ble *y). In the implementation, the variable definition task is accomplished in the local memory, which stores the value of $\left(t_{1}, t_{2}, t_{3} \ldots t_{n}\right)$. The main codes are described as follows.

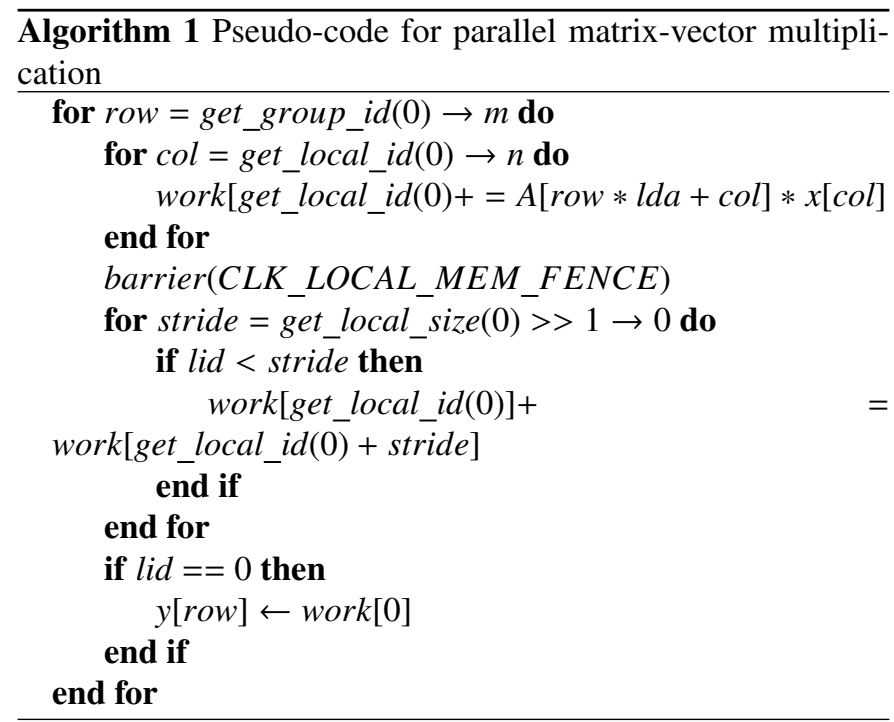

\subsection{Parallel Implementation of Matrix-matrix Multiplication}

The matrix-matrix multiplication task can be expressed as: $C=A B\left(\mathrm{C} \in R^{M * N}, \mathrm{~A} \in R^{M * P}, \mathrm{~B} \in R^{P * N}\right)$. The implementation of the serial program is defined mathematically as,

$$
C_{i, j}=C_{i, j}+\sum_{k=0}^{P} A_{i, k} * B_{k, j} \quad 0 \leq i \leq M, 0 \leq j \leq N .
$$

According to the equation above, the matrix $C$ must be calculated to access the row of matrix $A$ and column of matrix $B$ multiple times. If these data are stored directly in the global memory of the GPU, which has high access latency, it is necessary to load $2 * M * N * P$ times and to store $M * N$ times, which would lead to a low computational efficiency. However, the local memory of the GPU has low access latency and the size of the local memory is fixed in each work-group; therefore, it is better to use the matrix block method, i.e., matrix $A$ is divided into rows, matrix $B$ is divided into columns, and the block size is $B L O C K \_S I Z E^{*} B L O C K \_S I Z E$. The number of threads in a work-group is set as the block size. Thus, a thread processes a point in a sub-block and the threads in the work-group will process the points in the block size, as shown in Fig.5.

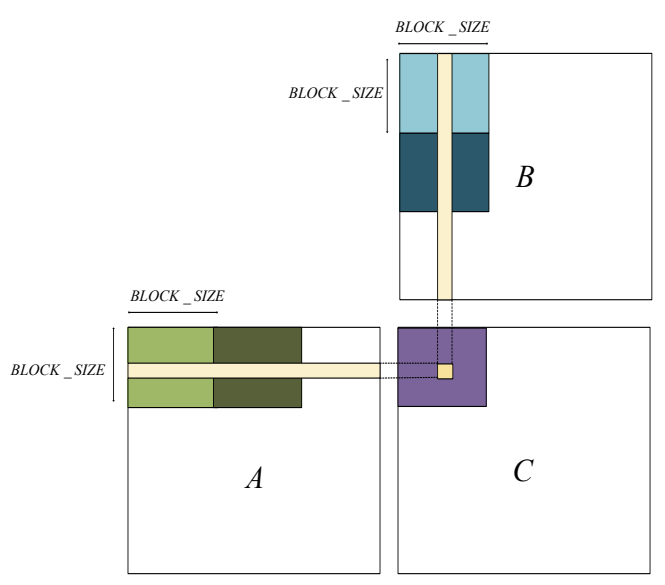

Figure 5: Parallel design of block matrix multiplication.

Using this method, the efficiency of matrix-matrix multiplication can be improved to a certain extent, but further optimization is necessary to improve its performance. For simplicity, let us assume that the matrix $A$ and matrix $B$ are each divided into blocks, i.e., matrix $A_{1}$ and matrix $B_{1}$. When we compute a point value for matrix $C_{1}$, it is necessary to access the local memory twice: once to read the corresponding values of $A_{1}$ and $B_{1}$, and once to calculate $C_{1}$. Thus, many local memory accesses are required to calculate matrix $C_{1}$. If a thread can handle this operation, it would reduce the local memory accesses and improve the algorithm's performance (Fig.6). First, we define the constant $T$ and the number of threads in a row of the working group is BLOCK_SIZE / T. Thus, we compute the value of the three elements in matrix $C_{1}$ via four local memory accesses and three calculations. Therefore, this can reduce the number of memory accesses compared with the former method.

Based on the parallel design described above, the coding work is described in the following. When the matrix $B$ in the global memory is copied to the local memory, it should be transposed to allow combined access. Thus, we first transpose matrix $B$. Depending on the performance of the GPU card, we set $T$ and BLOCK_SIZE accordingly. Next, we create the kernel function: kernel void MatrixMult (const int $m$, const int $n$ const, int $p$, _global const double * A, int lda, _global const double * B, int ldb, global double $* C$, int ldc). Assuming that $p$ is an integer multiple of BLOCK_SIZE, we define As and $B s$ as local variables to store the corresponding block data for matrix $A$ and $B$, respectively, and $a c c$ is a private variable, where the main code is given as follows. 


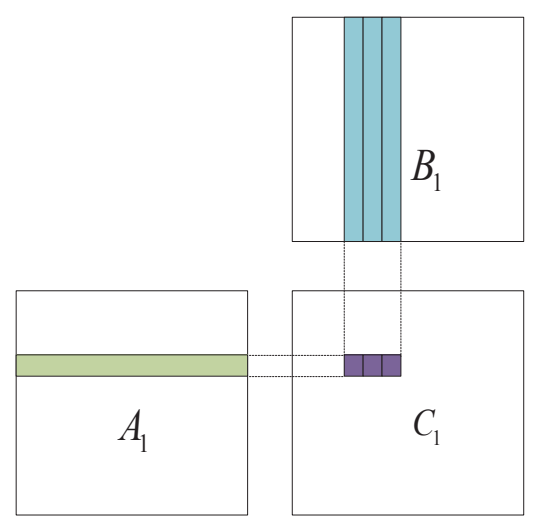

Figure 6: Optimization of the parallel design for block matrix multiplication.

\subsection{Parallel Implementation of Cholesky Factorization}

In the reconstruction algorithm, the estimation of the new signal is the most time-consuming step, which is usually performed with the least squares method. The least squares method can be formulated as: $x=\left(A^{T} A\right)^{-1} A^{T} y$. Assuming that $B=A^{T} A$ and $b=A^{T} y$, the equation can be translated into solving the following linear equation: $B x=b$. Many numerical methods are available for solving different types of linear equations, such as QR decomposition, LU decomposition, and Cholesky decomposition [31]. The matrix is a symmetric positive definite matrix, so the Cholesky decomposition method is selected because it is the most efficient method for handling symmetric positive definite matrices. The Cholesky decomposition method can be formulated as $B=L L^{T}$, where $L$ is the upper triangular matrix. During parallel Cholesky decomposition on a GPU, the standard Cholesky decomposition method has a very high data dependency and it is not suitable for parallel processing. Therefore, we use the block Cholesky decomposition method from the Lapack library [32]. The parallel Cholesky design is illustrated in Fig.7.

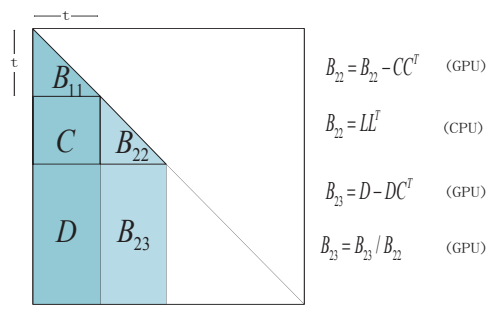

Figure 7: Parallel Cholesky decomposition.

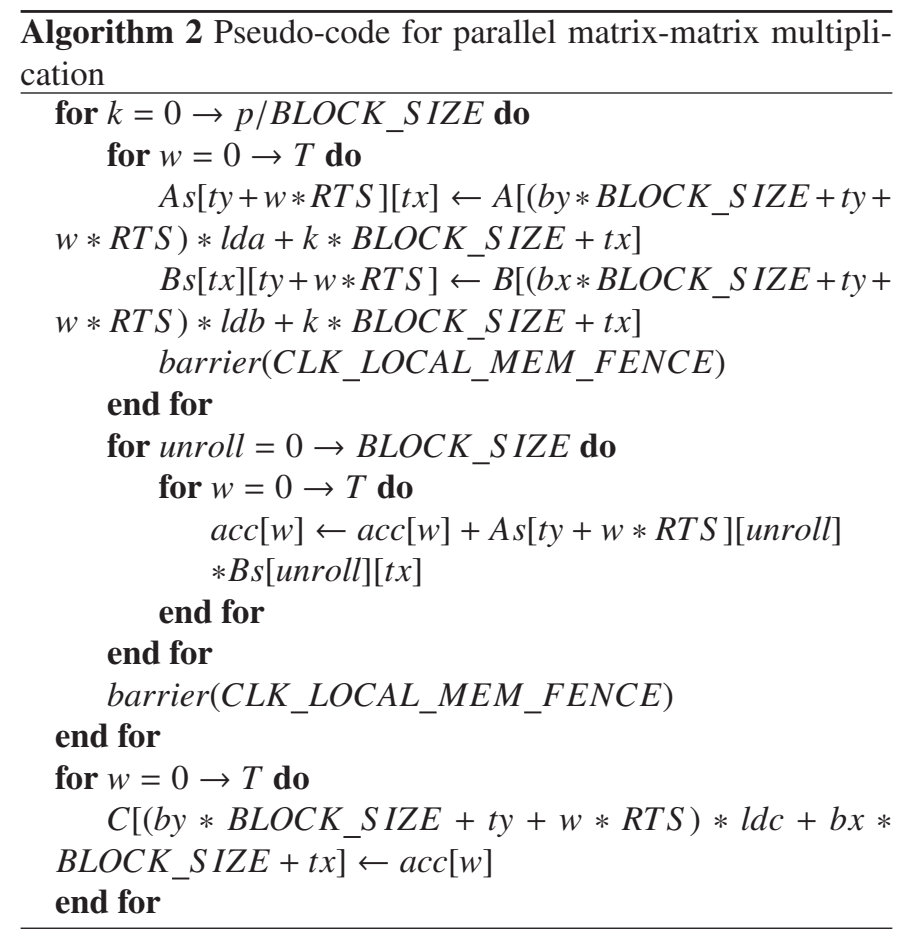

\section{Experiments and Analysis}

\subsection{Experimental Platforms}

In order to verify that the proposed parallel CoSaMP algorithm can run on different heterogeneous computing platforms and to assess its performance on different platforms, we performed experiments using two different GPU architectures: an 
Table 3: Configuration information for the two platforms.

\begin{tabular}{lcc}
\hline Platform & Hardware Configuration & Software Configuration \\
\hline \multirow{2}{*}{ AMD } & Intel(R) i5-3470 CPU @ 3.20GHZ & gcc-4.4.7 amdcl64.icd clblas-2.2.0 \\
& AMD Radeon HD 7350, 80 SMs & gdal-1.10.1 proj-4.8.0 geos-3.4.2 \\
\hline \multirow{2}{*}{ NVIDIA } & Intel(R) E5-2670 CPU @ 2.60GHZ & gcc-4.4.6 nvidia.icd clblas-2.2.0 \\
& Tesla K20Xm, 2688 SMs & gdal-1.10.1 proj-4.8.0 geos-3.4.2 \\
\hline
\end{tabular}

Table 4: Execution times for the serial and parallel CoSaMP algorithms on the AMD platform.

\begin{tabular}{lll}
\hline Image Size & Serial Time $(s)$ & Parallel Time $(s)$ \\
\hline $512 * 512$ & 27.19 & 12.54 \\
\hline $1024 * 1024$ & 412.54 & 37.69 \\
\hline $2048 * 2048$ & 6583.81 & 160.82 \\
\hline
\end{tabular}

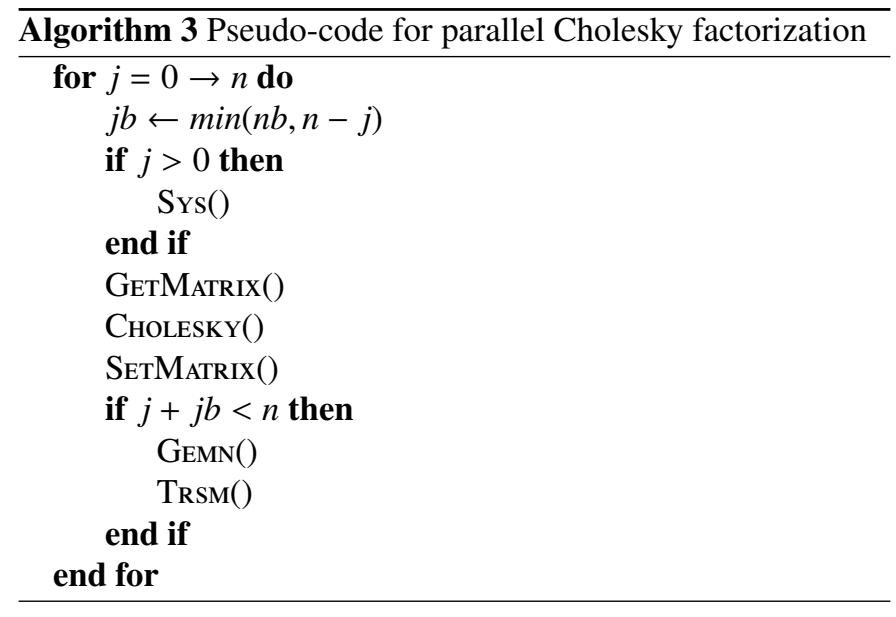

AMD GPU and an NVIDIA GPU. The AMD GPU was a traditional heterogeneous computing platform with an Intel CPU and an AMD GPU card. The NVIDIA platform was a dedicated high-performance heterogeneous computing platform equipped with an NVIDIA GPU card. The detailed configurations and the differences between the two platforms are shown in Table 3.

\subsection{Experimental Data}

The experimental datasets comprised RS images obtained from the Landsat-7 satellite. In order to compare the performance with different data sizes, three groups of datasets with image sizes of $512 * 512,1024 * 1024$, and $2048 * 2048$ were clipped from the original dataset with ENVI software. The datasets were in GeoTiff format. In addition, all of the parameters were set to the default values used for traditional processing.

\subsection{Performance Evaluation Index}

Speedup is a simple and direct performance evaluation index for assessing the processing efficiency of a parallel algorithm, which can provide users with a direct performance indicator. Thus, we used the speedup to evaluate the performance of the parallel CoSaMP algorithm. The speedup is defined as follows [33].

$$
S_{p}=T_{1} / T_{p}
$$

where $S_{p}$ is the speedup, $T_{1}$ is the execution time of the serial algorithm, and $T_{p}$ is the time required by the parallel program, and higher values of $S_{p}$ indicate better performance.

\subsection{Experimental Results and Analysis}

The implementation was tested using RS images with three different sizes and the sampling rate parameter was set to 0.5 . First, we recorded the running time required by the sequential CoSaMP algorithm on a personal computer using only the CPU, before testing the serial algorithm and its corresponding parallel algorithm on the platforms mentioned above, where we recorded the running time for $T_{p}$. Subsequently, we calculated the corresponding speed ratio with equation (5). In order to ensure that we obtained accurate estimates of the test time, the recording time was the mean value of three independent execution times.

\subsubsection{Experiments on the AMD GPU platform}

The running times required by the serial and parallel CoSaMP algorithms are given in Table 4.

As shown in Table 4, the processing time was reduced significantly by using the parallel CoSaMP algorithm compared with that for the serial algorithm on a single CPU. In addition, the reductions in the processing time differed according to the size of the input image. In this experiment, the reductions were $53.88 \%, 90.73 \%$, and $97.56 \%$ with image sizes of $512 * 512$, $1024 * 1024$, and $2048 * 2048$, respectively. According to equation (5), the speedups for these RS images with three different sizes are shown in Fig.8.

As shown in Fig.8, the speedup obtained increased with the input matrix size. Thus, the speedup obtained was not a stable amount and the maximum size of the image resulted in a larger increase in the ratio. This phenomenon was related to the capacity of the GPU hardware. When the image size was small, the speedup was relatively small, mainly because it could not fully saturate the pipelines to hide the latency. When the image size was $2048 * 2048$, the speedup was up to 41 times due to the higher GPU utilization ratio. In general, the experimental 


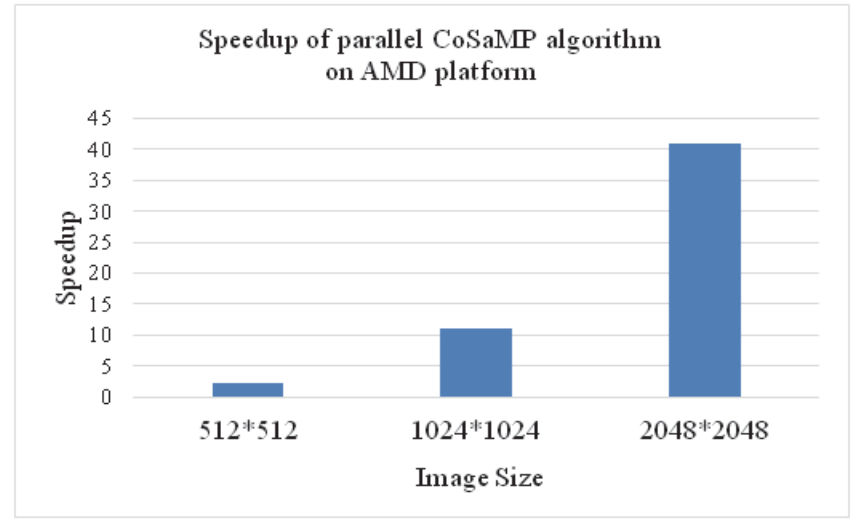

Figure 8: Speedup obtained with the parallel CoSaMP algorithm on the AMD platform.

results demonstrate that the parallel algorithm can greatly reduce the processing time for image reconstruction and obtain satisfactory performance as the image size increases.

\subsubsection{Experiments on the NVIDIA GPU platform}

OpenCL is a heterogeneous parallel language, so parallel algorithms implemented in OpenCL can be executed directly on different platforms. To verify its cross-platform capabilities, we used the same setup to test the parallel CoSaMP algorithm on the NVIDIA platform without any modifications. The results obtained using this platform are shown in Table 5.

As shown in Table 5, the processing time required for the parallel CoSaMP algorithm was reduced significantly on the NVIDIA platform compared with the serial algorithm, and thus our conclusions are similar to those based on Table 4. However, compared with serial processing, the time required for the parallel CoSaMP algorithm was reduced greatly on the NVIDIA platform, i.e., the reduction of the processing time was much higher on this platform. The reductions were $84.83 \%, 95.47 \%$, and $98.30 \%$ with image sizes of $512 * 512,1024 * 1024$, and $2048 * 2048$, respectively. Based on the results shown in Table 4 and Table 5, we can conclude that the proposed parallel algorithm delivers good performance and that it is portable across different computing platforms. According to equation (5), the obtained speedups are shown in Fig.9.

As shown in Fig.9, the speedup increased with the matrix size, which is similar to the trend in the speedup obtained using the parallel algorithm on the AMD platform. Thus, the speedup was lower when the image size was small, and the maximum speedup was obtained with an image size of 2048*2048, i.e., 58 times. Compared with the test results obtained using the AMD platform, the speedup was much higher on the NVIDIA platform. This is mainly because the computational capacity of NVIDIA K20XM was nearly 38 times that of AMD HD 7350.

The results of these experiments demonstrate that the parallel CoSaMP algorithm can speed up the processing time for image reconstruction, as well as enhancing the cross-platform portability, thereby avoiding the limitations of a parallel algorithm developed to target a single computational platform (e.g.,

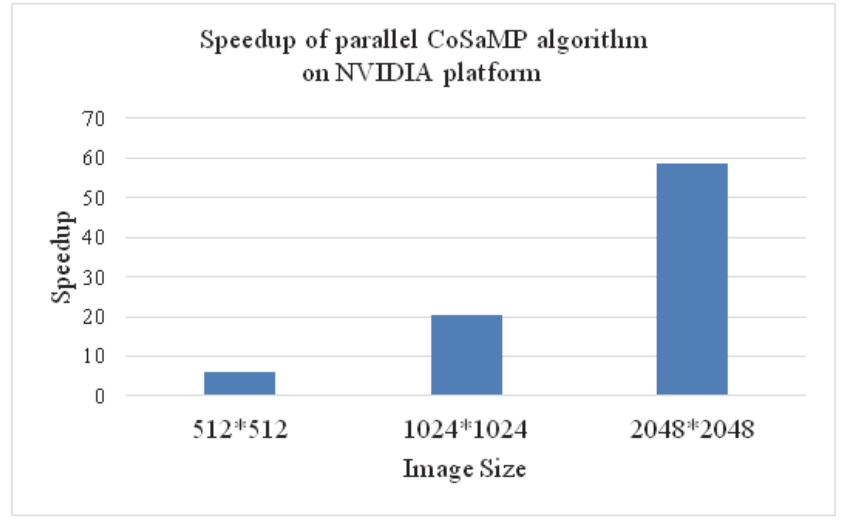

Figure 9: Speedup using the parallel CoSaMP algorithm on the NVIDIA platform.

a parallel algorithm constructed with CUDA can only run on NVIDIA hardware).

\section{Conclusion and Future Work}

In this study, we described the design and implementation of a parallel CoSaMP algorithm using an OpenCL programming model on GPU platforms to reduce the processing time for the reconstruction algorithm in CS. According to various tests, we demonstrated that our parallel algorithm could run on both the AMD and NVIDIA platforms without any modifications, and its performance was also maintained.In addition, better performance was obtained when the processed images were larger in size. According to our experiments, the maximum speedup rates were 41 times and 58 times with an image size of $2048 * 2048$ on AMD GPU and NVIDIA K20XM cards, respectively.

The parallel algorithm can also be improved further. For example, both experimental platforms are equipped with CPUs and GPU, but the CPU was almost in an idle state in our experiments. Thus, the expected overall performance would be better if some calculation tasks could be assigned to the CPU. According to our preliminary experiments using a parallel universal Kriging algorithm on CPU+GPU/CPU+MIC platforms with a simple scheduling strategy, the speedup increased slightly when the CPU computing resources were also utilized. This is because the computing capacity of the CPU is relatively low compared with that of the GPUs. We are currently considering various dynamic load balancing strategies[? ] to fully exploit multi-threading techniques on the CPU and GPU, thereby improving the performance of parallel algorithms constructed with OpenCL.

\section{Acknowledgments}

This study was supported mainly by the National Natural Science Foundation of China (Grant Nos. 41001221, 51277167, 41471368, and 41571413); the Fundamental Research Funds for the Central Universities (Grant No. ZYGX2015J111); the 
project entitled "Design and development of the parallelism for typical remote sensing image algorithm based on heterogeneous computing" from the Institute of Remote Sensing and Digital Earth, Chinese Academy of Sciences; and also the National Science Foundation of the United States (Award No. 1251095). This study used computing resources provided by LSU/LONI and we wish to thank the support staff for their hard work and support.

\section{References}

[1] D. L. Donoho, "Compressed sensing," Information Theory, IEEE Transactions on, vol. 52, no. 4, pp. 1289-1306, 2006.

[2] R. G. Baraniuk, "Compressive sensing," IEEE signal processing magazine, vol. 24, no. 4, pp. 118-121, 2007.

[3] Q. Bai and C. Jin, "Image fusion and recognition based on compressed sensing theory," International Journal on Smart Sensing and Intelligent Systems, vol. 8, no. 1, pp. 159-180, 2015.

[4] C. Pohl and J. L. V. Genderen, "Review article multisensor image fusion in remote sensing: concepts, methods and applications," International Journal of Remote Sensing, vol. 19, no. 5, pp. 823-854, 1998.

[5] A. Maleki and D. L. Donoho, "Optimally tuned iterative reconstruction algorithms for compressed sensing," IEEE Journal of Selected Topics in Signal Processing, vol. 4, no. 2, pp. 330-341, 2010.

[6] W. Xue, C. Yang, H. Fu, X. Wang, Y. Xu, J. Liao, L. Gan, Y. Lu, R. Ranjan, and L. Wang, "Ultra-scalable CPU-MIC acceleration of mesoscale atmospheric modeling on tianhe-2," IEEE Trans. Computers, vol. 64, no. 8, pp. 2382-2393, 2015.

[7] S. Meng, W. Dou, X. Zhang, and J. Chen, "KASR: A keyword-aware service recommendation method on mapreduce for big data applications," IEEE Trans. Parallel Distrib. Syst., vol. 25, no. 12, pp. 3221-3231, 2014.

[8] C. Liu, J. Chen, L. T. Yang, X. Zhang, C. Yang, R. Ranjan, and K. Ramamohanarao, "Authorized public auditing of dynamic big data storage on cloud with efficient verifiable fine-grained updates," IEEE Trans. Parallel Distrib. Syst., vol. 25, no. 9, pp. 2234-2244, 2014.

[9] X. Wu and J. Deng, "Compressed sensing parallel processing algorithm based on openmp," Journal of Computer Applications, vol. 32, no. 3, pp. 617-619, 2012.

[10] Y. Zhang and G. Zhang, "Mass data processing in internet of things based on parallel compressed sensing," Computer Applications $\mathcal{E}$ Software, vol. 29, no. 10, pp. 58-61, 2012.

[11] D. Yang, H. Li, G. D. Peterson, and A. Fathy, "Compressed sensing based uwb receiver: Hardware compressing and fpga reconstruction." in Information Sciences and Systems, 2009. CISS 2009. 43rd Annual Conference on, 2009, pp. 198-201.

[12] J. Chen, J. Cong, M. Yan, and Y. Zou, "Fpga-accelerated 3d reconstruction using compressive sensing." in Proceedings of the ACM/SIGDA international symposium on Field Programmable Gate Arrays, 2012, pp. 163-166.

[13] J. D. Owens, L. David., and G. Naga., "A survey of general-purpose computation on graphics hardware," Computer Graphics Forum, vol. 26, no. 1, p. 80ÅC113, 2007.

[14] Y. Zou, W. Xue, and S. Liu, "A case study of large-scale parallel I/O analysis and optimization for numerical weather prediction system," Future Generation Comp. Syst., vol. 37, pp. 378-389, 2014.

[15] L. Gan, H. Fu, W. Luk, C. Yang, W. Xue, X. Huang, Y. Zhang, and G. Yang, "Solving the global atmospheric equations through heterogeneous reconfigurable platforms," TRETS, vol. 8, no. 2, p. 11, 2015.

[16] L. Li, W. Xue, R. Ranjan, and Z. Jin, "A scalable helmholtz solver in GRAPES over large-scale multicore cluster," Concurrency and Computation: Practice and Experience, vol. 25, no. 12, pp. 1722-1737, 2013.

[17] A. Borghi, J. Darbon, Peyronnet, S. Peyronnet, T. F. Chan, and S. Osher, "A simple compressive sensing algorithm for parallel many-core architectures," CAM Report, pp. 08-64, 2008.

[18] M. Andrecut, "Fast gpu implementation of sparse signal recovery from random projections." Engineering Letters, vol. 17, no. 17, pp. 151-158, 2008.

[19] Y. Fang, L. Chen, J. Wu, and B. Huang, "Gpu implementation of orthogonal matching pursuit for compressive sensing," in Parallel and Distributed
Systems (ICPADS), 2011 IEEE 17th International Conference on, 2011, pp. 1044-1047.

[20] M. M. Geng, C. L. Jiang, and B. C. Zhang, "Parallel implementation of iterative shrinkage-thresholding algorithm via cuda," Journal of University of Chinese Academy of Sciences, vol. 30, no. 5, pp. 676-681, 2013.

[21] J. D. Blanchard and J. Tanner, "Performance comparisons of greedy algorithms in compressed sensing," Numerical Linear Algebra with Applications, vol. 22, no. 2, pp. 254-282, 2015.

[22] G. Chen, Shuai Li and H. Zhang, "A gpu-based parallel implementation of compressive sampling reconstruction for sar image compression," $D i$ anzi Yu Xinxi Xuebao/journal of Electronics E Information Technology, vol. 33, no. 3, pp. 610-615, 2011.

[23] J. Nickolls, I. Buck, M. Garland, and K. Skadron, "Scalable parallel programming with cuda," in ACM SIGGRAPH 2008 classes, 2008, pp. 4053.

[24] J. E. Stone, D. Gohara, and G. Shi, "Opencl: A parallel programming standard for heterogeneous computing systems," Computing in Science $\mathcal{E}$ Engineering, vol. 12, no. 3, pp. 66-73, 2010.

[25] J. Lu, H. Zhang, and H. Meng, "Novel hardware architecture of sparse recovery based on fpgas," in Signal Processing Systems (ICSPS), 2010 2nd International Conference on, 2010, pp. V1-302-V1-306.

[26] D. Needell and J. A. Tropp, "Cosamp: Iterative signal recovery from incomplete and inaccurate samples," Applied $\mathcal{E}$ Computational Harmonic Analysis, vol. 26, no. 12, pp. 93-100, 2008.

[27] M. Pharr and R. Fernando, "Gpu gems 2: Programming techniques for high-performance graphics and general-purpose computation (gpu gems),".G688 2005, 2005.

[28] J. Nickolls and W. J. Dally, "The gpu computing era," IEEE Micro, vol. 30, no. 2, pp. 56-69, 2010.

[29] S. S. Baghsorkhi and M. Delahaye, An adaptive performance modeling tool for GPU architectures. ACM, 2010.

[30] M. I. Gordon, W. Thies, and S. Amarasinghe, "Exploiting coarse-grained task, data, and pipeline parallelism in stream programs," Acm Sigarch Computer Architecture News, vol. 41, no. 11, pp. 151-162, 2006.

[31] H. Harbrecht, M. Peters, and R. Schneider, "On the low-rank approximation by the pivoted cholesky decomposition," Applied Numerical Mathematics, vol. 62, no. 4, p. 428ÅC440, 2012.

[32] E. Anderson, Z. Bai, J. Dongarra, and Greenbaum, "Lapack: a portable linear algebra library for high-performance computers," in Proceedings of the 1990 ACM/IEEE conference on Supercomputing. IEEE Computer Society Press, 1990, pp. 2-11.

[33] S. Brawer, Introduction to parallel computing. San Diego,CA: Academic Press, 1989. 
Table 5: Execution times for the serial and parallel CoSaMP algorithms on the NVIDIA platform.

\begin{tabular}{lll}
\hline Image Size & Serial Time $(\mathrm{s})$ & Parallel Time $(\mathrm{s})$ \\
\hline $512 * 512$ & 35.13 & 5.33 \\
\hline $1024 * 1024$ & 528.82 & 23.97 \\
\hline $2048 * 2048$ & 7887.84 & 133.18 \\
\hline
\end{tabular}


Fang Huang is an associate professor of School of Recourses and Environment (SRE) in University of Electronic Science and Technology of China (USTEC). He received his doctor degree of High Performance Geo-computation (HPGC) from the Institute of Remote Sensing Applications (IRSA), Chinese Academy of Science (CAS). He received the B.S. degree of surveying and mapping Engineering in Taiyuan University of Technology (TUT), Taiyuan, China, the M.S. in photogrammetry and remote sensing from Beijing Jiaotong University (BJTU), Beijing, China, in 2002 and 2005, respectively. His current research interests focus on putting the cut-edge computing techniques, e.g. the parallel computing, grid computing, cloud computing, and even heterogeneous computing into RS/GIS algorithms' designing and implementation.

Jian Tao is a research scientist / IT consultant at the Center for Computation \& Technology at Louisiana State University. He received his Ph.D. in computational astrophysics from Washington University in St. Louis in 2008. He got his B.S. on space physics from University of Science \& Technology of China (USTC) in 2000. His research interests include high performance computing, heterogeneous computing, computational framework, cyberinfrastructure, and numerical modeling.

Dr. Liu currently is an associate professor at the Institute of Remote Sensing and Digital Earth, Chinese Academy of Sciences. He received the M.S. degree in 2004 and the Ph.D. degree in 2009, both in signal processing, from Chinese Academic of Science. From May 2012 to May 2013, he was with Department of Electrical and Computer Engineering, George Washington University as a Visiting Scholar. He has published 30+ scientific, peer-reviewed papers. He is currently an associate editor of Frontiers in Environmental Science. He is also the reviewer for Applied Remote Sensing, IEEE JSTAR, Neurocomputing, Signal Processing, etc. His research is focused on big data, sparse representation, compressive sensing, deep learning and their applications to remote sensing data processing.

Yang Xiang received the Ph.D. degree in computer science from Deakin University, Victoria, Australia. He is the Director of the Centre for Cyber Security Research, Deakin University, and the Associate Head of School (Industry Engagement), School of Information Technology. His research interests include network and system security, distributed systems, and data analytics. He has published more than 200 research papers in international journals and conferences. He serves as an Associate Editor of the IEEE TRANSACTIONS ON COMPUTERS, IEEE TRANSACTIONS ON PARALLEL AND DISTRIBUTED SYSTEMS, Security and Communication Networks, and an Editor of the Journal of Network and Computer Applications. He is a Senior Member of the IEEE.

Dr. Wang is a "ChuTian" Chair Professor at School of Computer Science, China Univ. of Geosciences (CUG), and a Professor at Inst. of Remote Sensing \& Digital Earth, Chinese Academy of Sciences (CAS). Prof. Wang received B.E. \& M.E from Tsinghua Univ. and Doctor of Eng. from Univ. Karlsruhe (Magna Cum Laude), Germany. Prof. Wang is a Fellow of IET, Fellow of British Computer Society. Prof. Wang serves as an Associate Editor of IEEE Tran. Computers and IEEE Tran. on Cloud Computing. His main research interests include high performance computing, e-Science, and spatial data processing. 

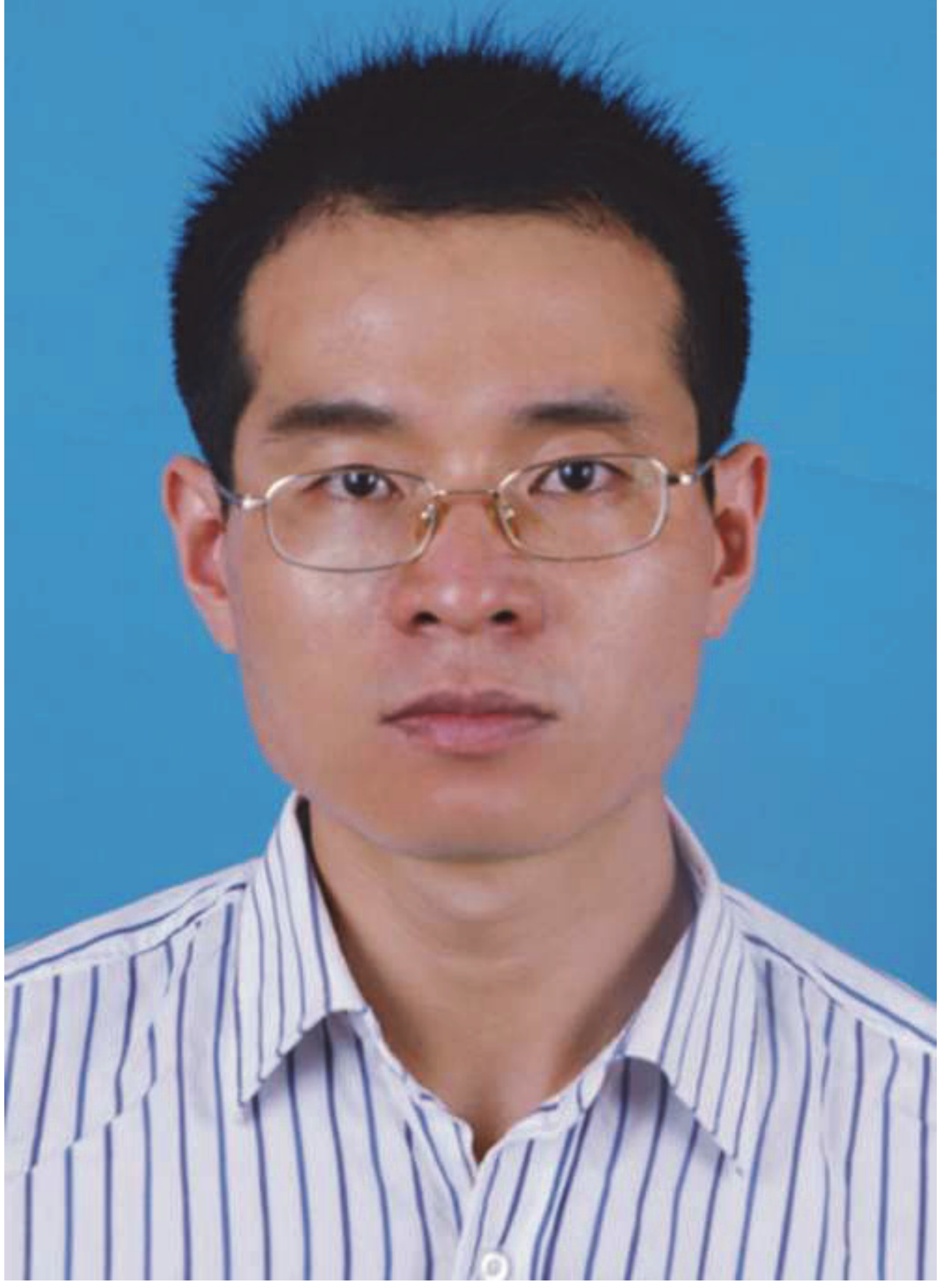


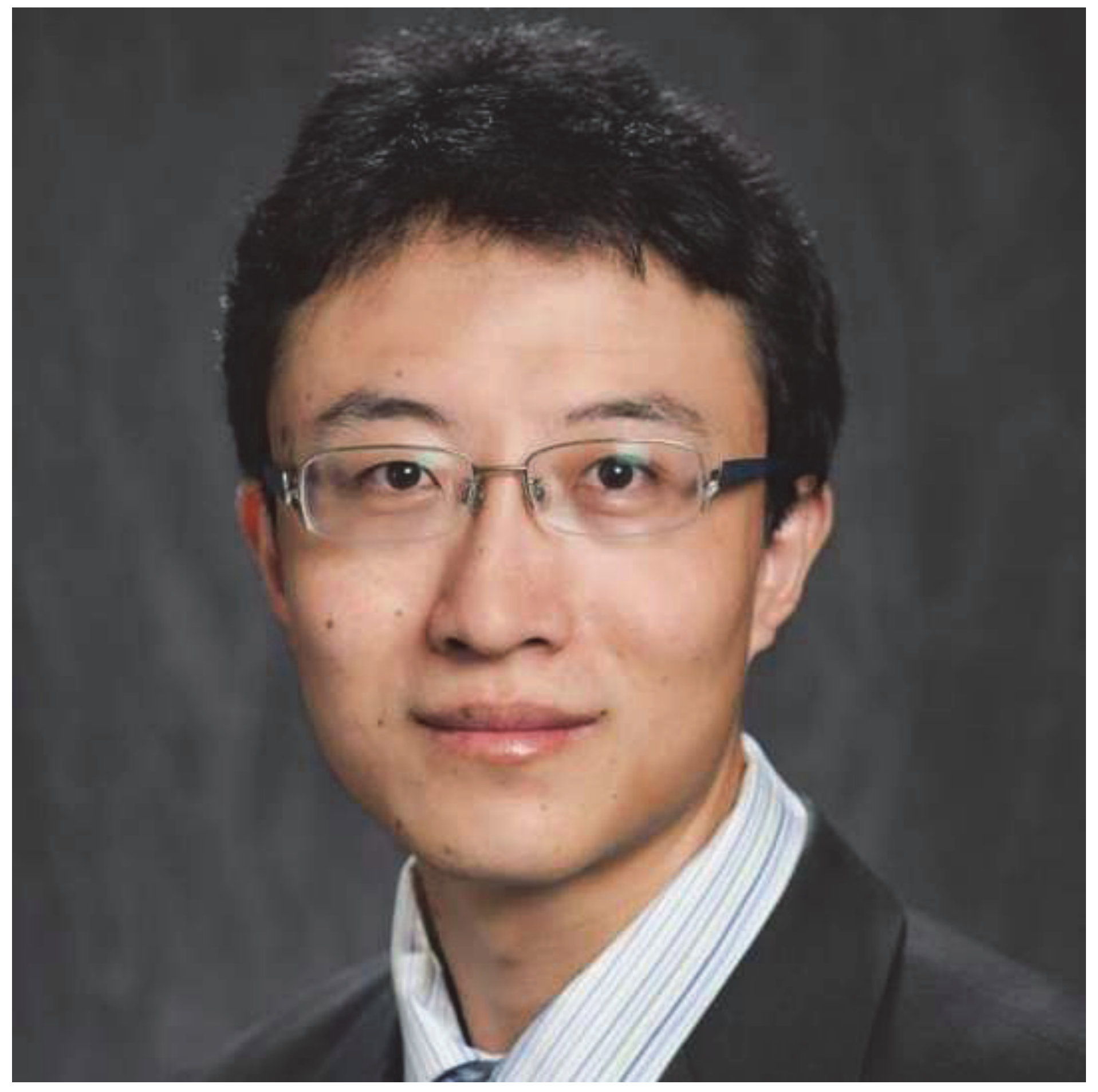




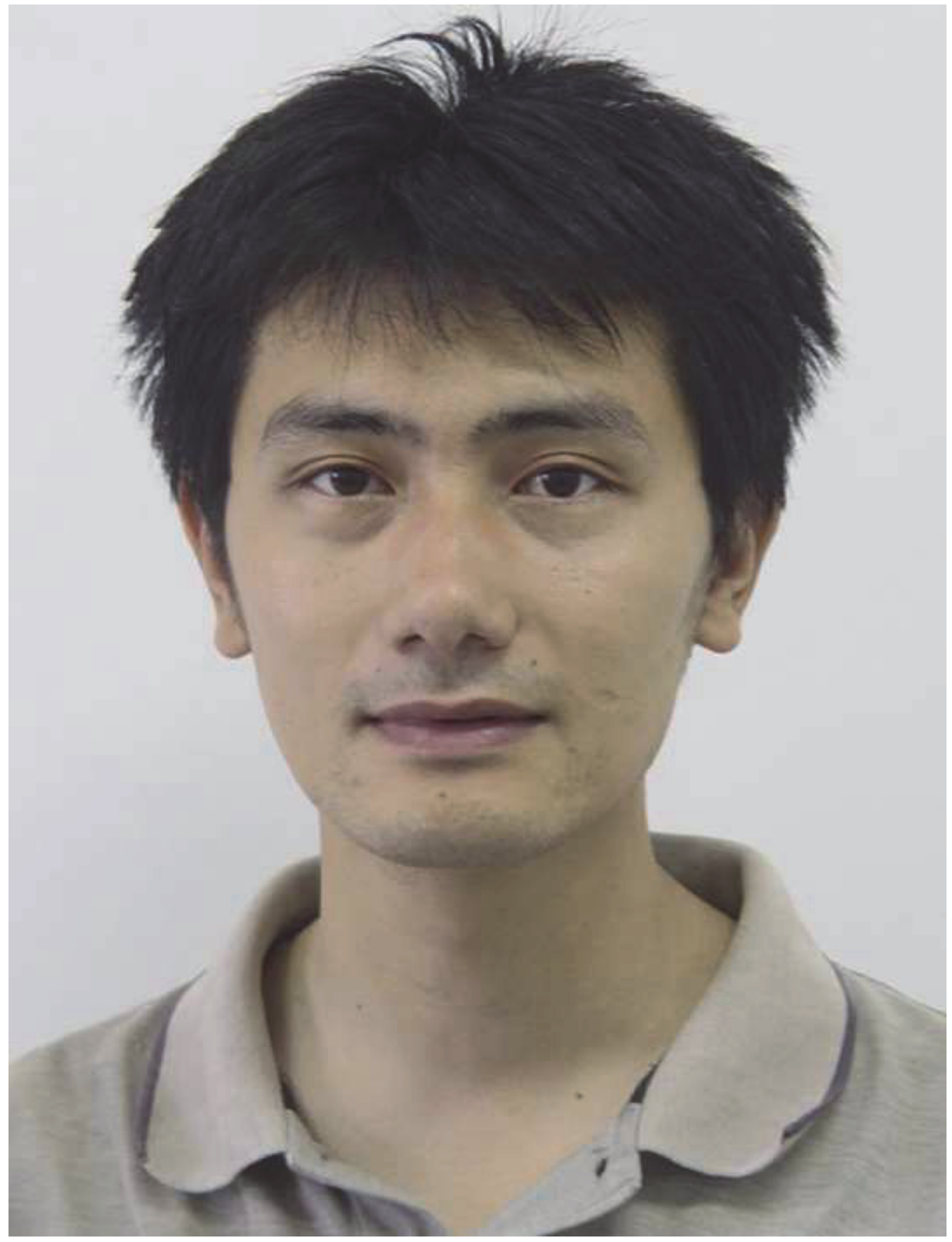




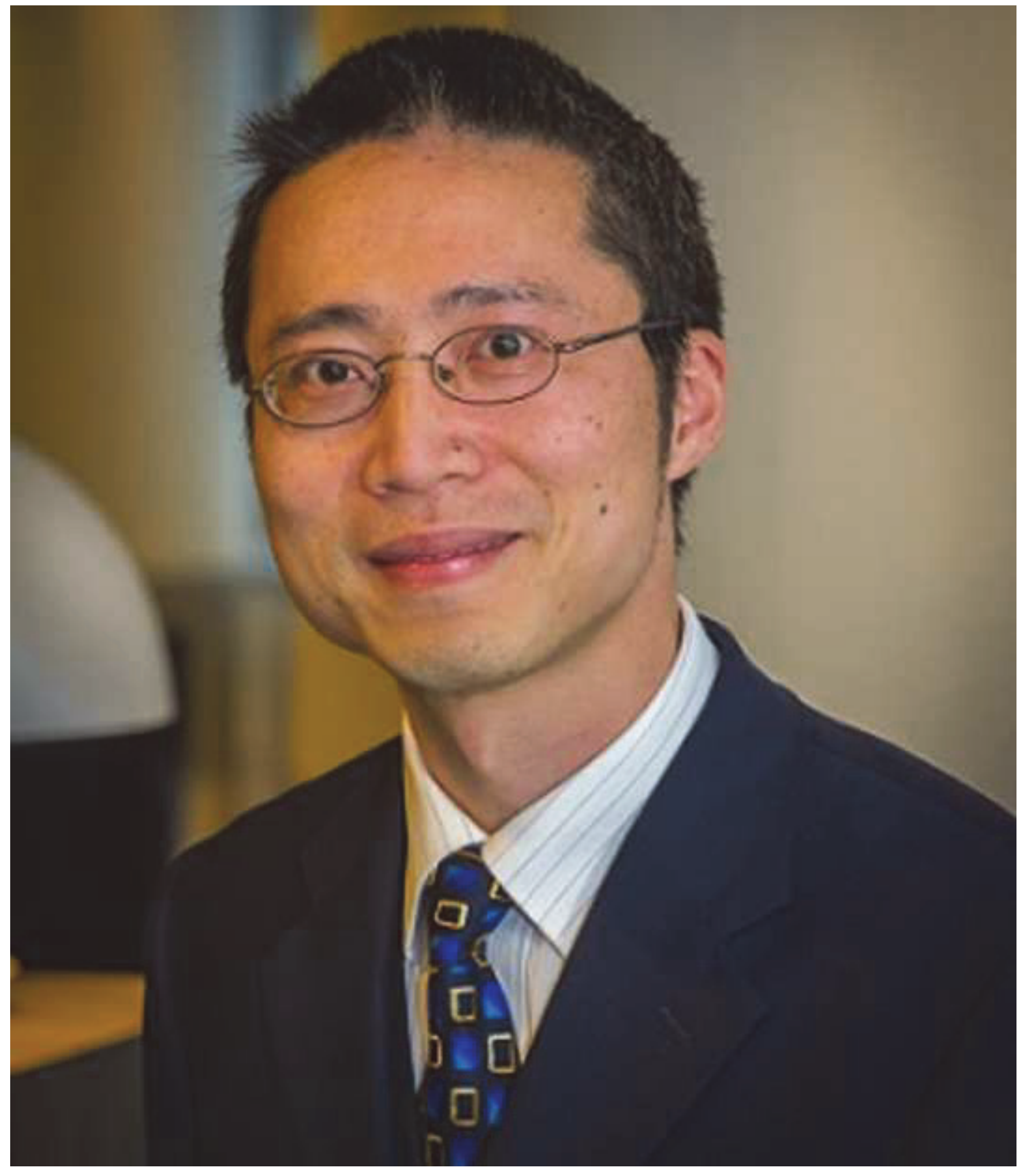




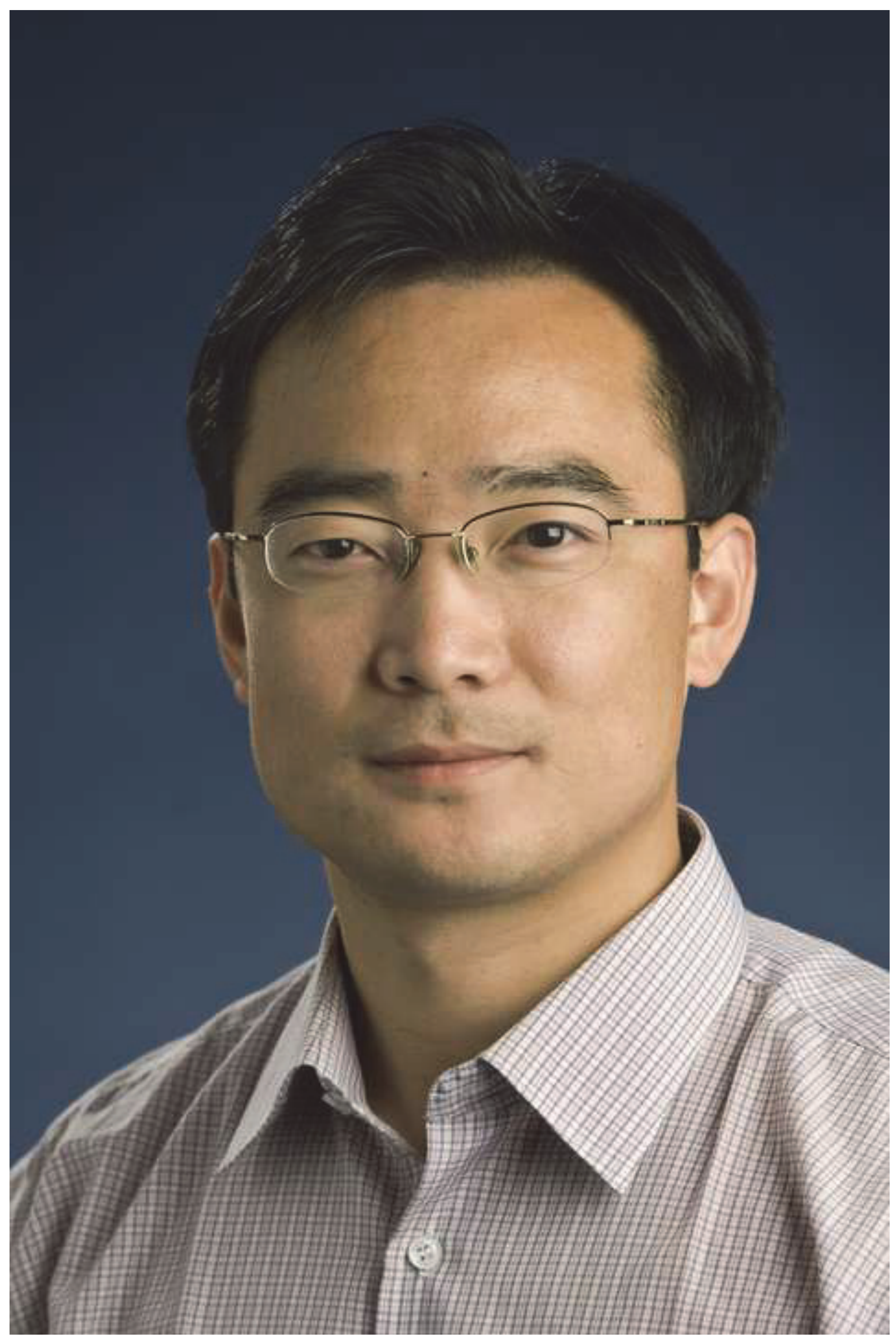




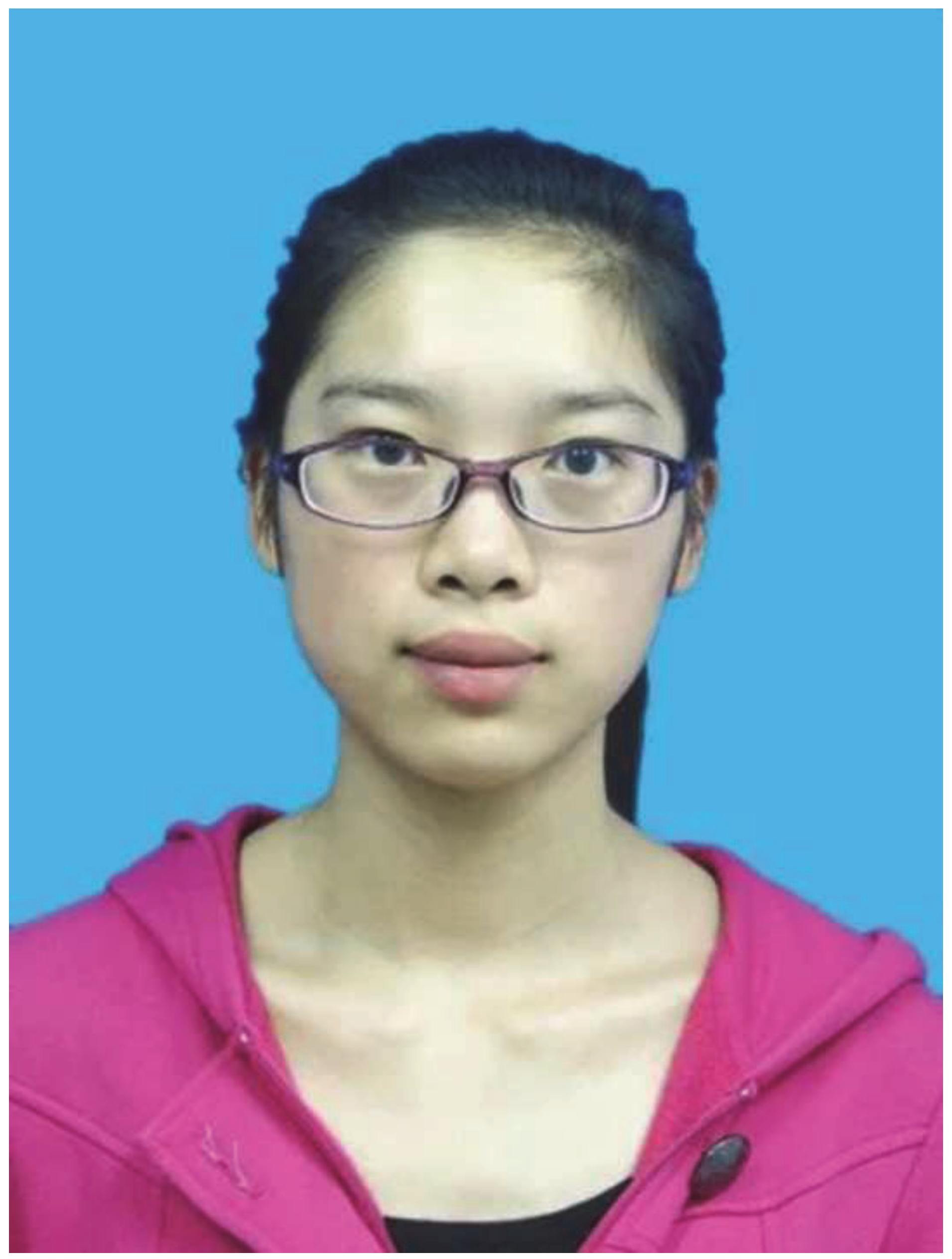

